\section{Semes for analysis of evolution: de Duve's peroxisomes and Meyer's hydrogenases in the sulphurous Proterozoic eon}

\section{Lynn Margulis, Michael Chapman and Michael F. Dolan}

Although de Duve's Perspective, “The origin of eukaryotes: a reappraisal" is masterful and relevant, certain lesser known but important work was overlooked. Support for his idea abounds: phagocytotic intracellular motility preceded the 'adoption' of mitochondria and plastids (FIG. 1). What de Duve calls "coherent collections of enzymes" are 'semes', the units of evolutionary analysis 2 .

The archaebacterial-eubacterial merger (Gupta's chimaera $^{3}$ ) resulted in membrane fusion; archaebacterial lipids and proteins formed the endoplasmic reticulum whereas the Golgi components evolved from eubacterial membrane biosynthesis ${ }^{4}$. The archaebacterial-eubacterial symbiotic merger of a thermoacidophilic sulphidogenic heterotroph ${ }^{5,6}$ with a motile sulphide-to-sulphur oxidizing heterotroph occurred under the threat of oxygen toxicity. From this syntrophy, hundreds of protists evolved. Their descendants thrive in anoxic habitats (for example, pelomyxids, mastigamoebae, devescovinids, oxymonads, trichomonads and other parabasalids). The presence of phagocytosis, mitosis and endocytosis in these amitochondriates testifies to the evolution of cytoskeletal motility (FIG. 2) before mitochondria ${ }^{2}$. The contributor of motility to the chimaera was the ancestral 'Perfil'ieva', a free-living, aerotolerant, sulphurous mud-scum-mat Spirochaeta-like eubacterium of geochemical significance $^{7,8}$ (now banked in the German culture collection in Braunschwieg, with strain accession numbers Str. P=DSMZ 19205 and Str. SR=DSMZ19230). By use of Hall's new algorithm, more than 50 genes for the synthesis of eukaryotic enzymes and lipids were acquired by the chimeric eukaryotes in the transition from a Spirochaeta-like eubacterium to the $[9(2)+2]$ motility organelle (J. L. Hall \& L.M., unpublished observations). At lower stringency, even more eukaryotic sequences in Perfilieva should be detected.

Permanent bacterial conjugationnucleoid membrane formation ${ }^{9}$ generated the nucleus tethered to $[9(2)+2]$ motility organelles (such as undulipodia including cilia) and its attachment apparatus, which became the centriole-centrosome system (Mixotricha is analogous ${ }^{10}$ ). The intron-less eubacterial DNA (now in the nucleolinus of the nucleolus) became the centrosomecentriole DNA ${ }^{11}$. Specific centrosomal RNAs and proteins for assembly and maintenance of the centriole-centrosome system ${ }^{12}$ are discussed in REF. 13. Redundancy reduction followed fusion. Genetic and metabolic systems acquired from intracellular motile symbionts were integrated and redeployed (comparable with what occurred in the evolution of Staurojoenina ${ }^{14}$, Peridinium balticum, Mesodinium rubrum and Hatena) ${ }^{15,16}$. Natural selection in microoxic habitats maintained heterotrophic chimaeras.

Information-molecule loss from centriole-kinetosomes (such as gene loss in plastids and mitochondria) occurred in the sulphurous Proterozoic eon (2500-541 million years $\mathrm{ago}^{17}$ ) during which time peroxisomes were acquired. Besides peroxisomes, organelles that are probably of bacterial origin that continued the trend of loss of genes to the nucleus until completion include some hydrogenosomes, $\gamma$-particles of Blastocladiella, and mitosomes.

Genome analysis of hydrogenase distribution is explicable only if hydrogen gas production entered anaerobic protists via at least two distinct events ${ }^{18}$ : acquisition of a cytosolic enzyme complex or of a symbiotic

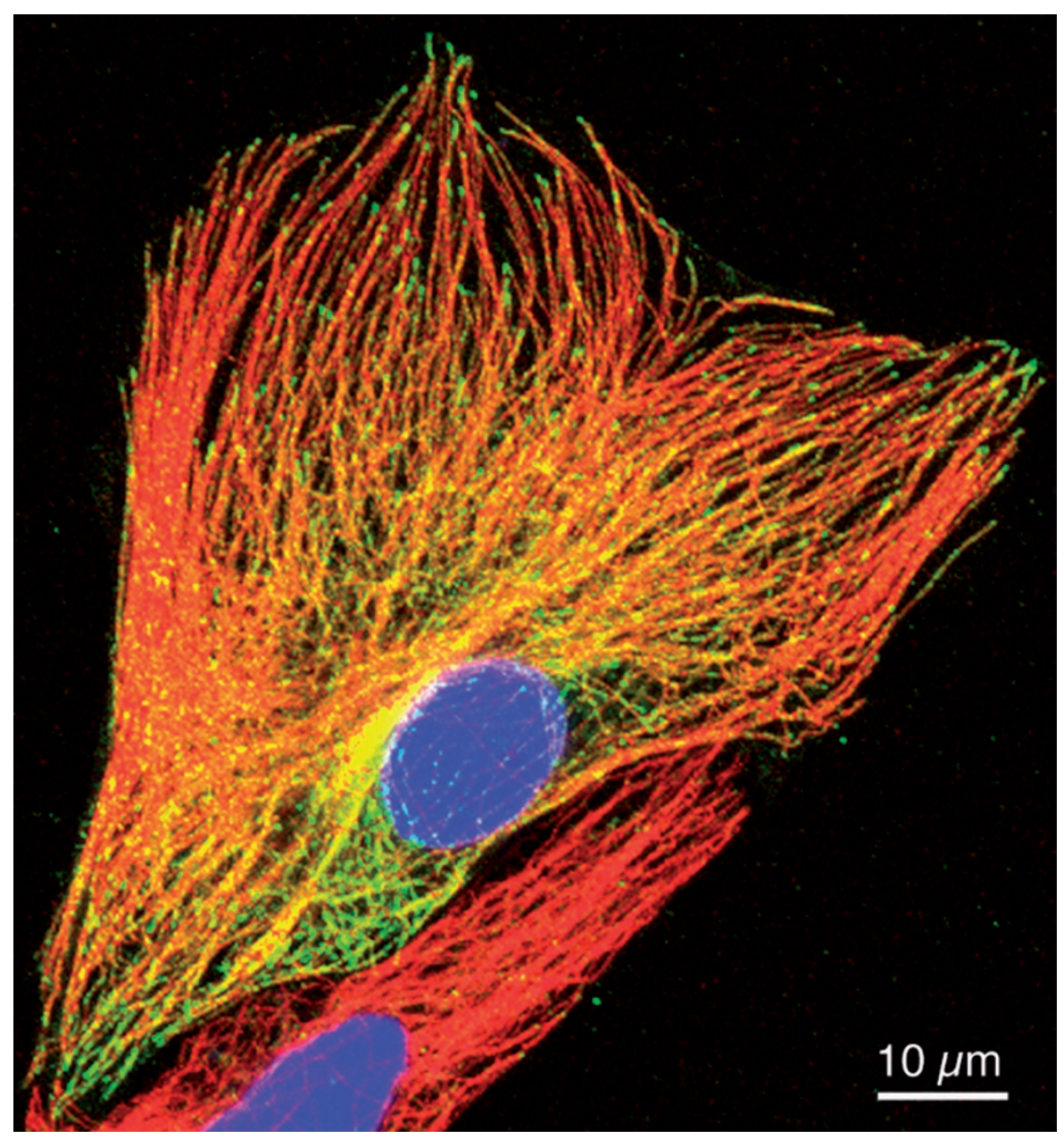

Figure 1 | Did this cell originate from an archaebacterium? The image shows a porcine epithelial cell (line LLCPK), with microtubules stained in red and the microtubule tip-binding protein EB1 stained in green. The nucleus is stained in blue. Image courtesy of Lynne Cassimeris, Department of Biology, Lehigh University, Bethlehem, Pennsylvania, USA. 


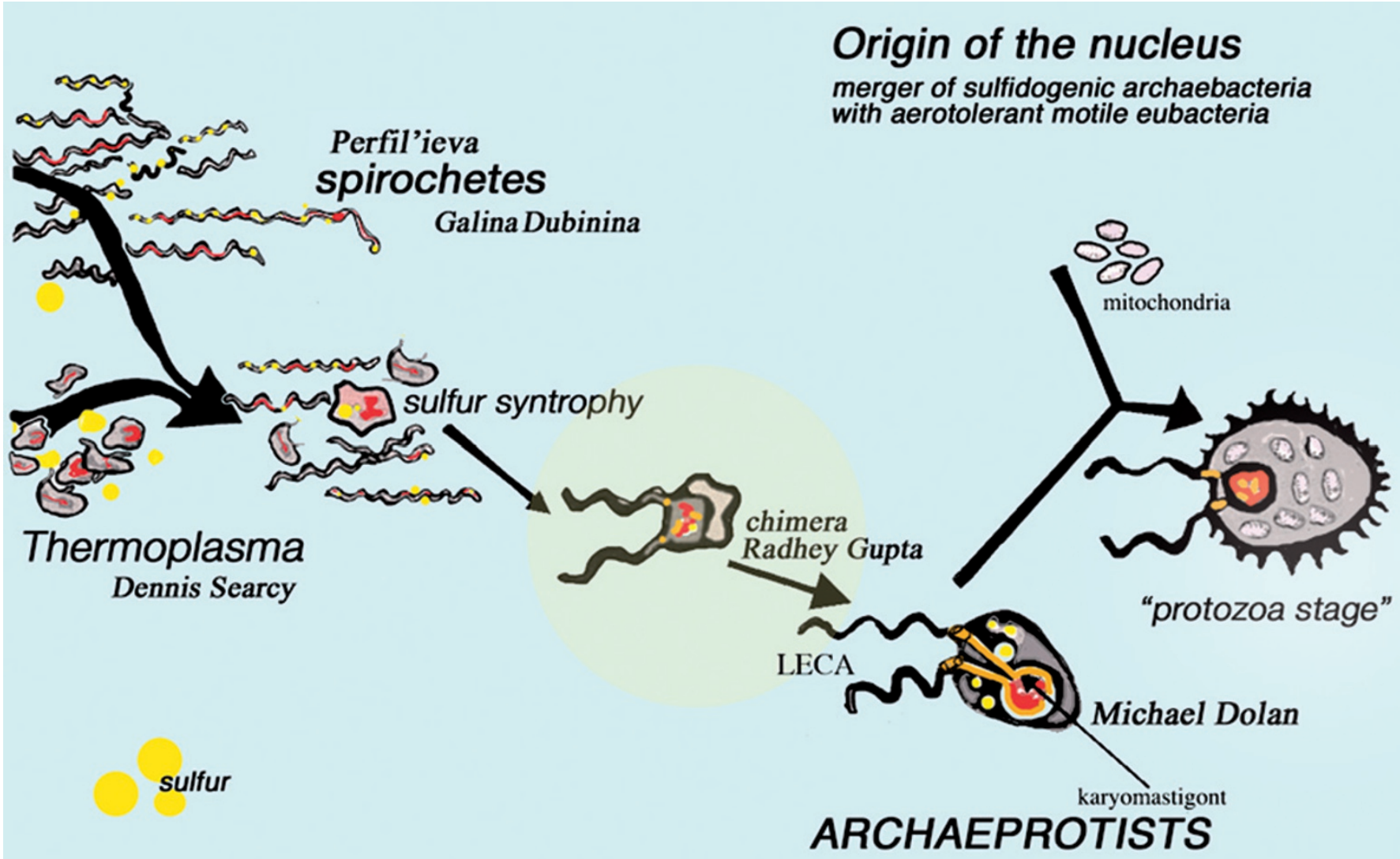

Figure 2 | Extant microbes exemplify each step in this karyomastigont model of the origin of the nucleocytoskeleton.

bacterium (or both). The hydrogenase seme derives from the chimaera's eubacterial partner (aerotolerant sulphide-oxidizing Perfil'ieva) or some other 'adopted' eubacterium. The hydrogenosomal Fe-Fe hydrogenases - for example, those of Trichomonas vaginalis and most parabasalids (many of which are multinucleate, but none of which are mitochondriate) - evolved from Clostridium-like bacteria. But the hydrogenases of Spironucleus, Giardia and Entamoeba histolytica are cytosolic; presumably they retained enzymes from the eubacterial ancestor (for example, the sulphur syntrophic Perfil'ieva). Anaerobic chytrids, even in the same genus, differ markedly (Neocallimastix ovalis versus Neocallimastix frontalis), confirming hydrogenase-hydrogenosome polyphyly ${ }^{19}$. The origin of $\mathrm{Fe}-\mathrm{Fe}$ hydrogenases that are incapable of generating hydrogen gas in 'crown taxa eukaryotes' (animals, plants and fungi) noted by Meyer ${ }^{17}$ is implied by the data that de Duve discussed. The eubacterial cytosolic or periplasmic hydrogenase complex that was acquired from eubacterial ancestors changed during eukaryosis as intracellular motility evolved in amitochondriates. The hydrogenase system hypertrophied, mutated or was lost in response to the rising oxygen threat. Dispensable hydrogen gas production was not selected for, but rather hydrogenases and/or hydrogenosomes and their components were retained for myriad other semes.

Semes must be identified. Amino-acid or nucleotide homologies without seme identification lead to systematic inaccuracy in evolutionary reconstruction. Molecular sequencing techniques may resolve origins, but not in absence of the knowledge of whole organisms in their paleoenvironments.

Department of Geosciences, Morrill Science Center, University of Massachusetts, Amherst, Massachussetts 01003, USA. Correspondence to L.M. e-mail: lynn@sagantechnology.com

doi: $10.1038 / \mathrm{nrg} 2071-\mathrm{c} 1$

de Duve, C. The origin of eukaryotes: a reappraisal. Nature Rev. Genet. 8, 395-403 (2007).

Margulis, L., Chapman, M., Guerrero, R. \& Hall, J. The last eukaryotic common ancestor (LECA): acquisition of cytoskeletal motility from aerotolerant spirochetes in the Proterozoic eon. Proc. Natl Acad. Sci. USA 103, 13080-13085 (2006).

Gupta, R. S. in Microbial Phylogeny and Evolution: Concepts and Controversies (ed. Sapp, J.) 261-280 (Oxford Univ. Press, New York, 2005)

Helenius, A. \& Aebi, M. Intracellular functions of $\mathrm{N}$-linked glycans. Science 291, 2364-2369 (2001). Searcy, D. G. Metabolic integration during the evolutionary origin of mitochondria. Cell Res. 13, 229-238 (2003)
Searcy, D. G. \& Lee. S. H. Sulfur reduction by human erythrocytes. J. Exp. Zool. 282, 310-322 (1998). Dubinina G. A Grabovich, M. Y \& Leshcheva, N. V. Occurrence, structure, and metabolic activity of Thiodendron sulfur mats in various saltwater environments. Microbiol. 62, 450-456 (1993)

8. Dubinina, G. A., Leshcheva, N. V. \& Grabovich, M. Y The colorless sulfur bacterium Thiodendron is actually a symbiotic association of spirochetes and sulfidogens. Microbiol. 62, 432-444 (1993).

9. Fuerst, J. A. \& Webb, R. I. Membrane-bounded nucleoid in the eubacterium Gemmata obscuriglobus. Proc. Natl Acad. Sci. USA 88, 8184-8188 (1991).

10. König, H. et al. The flagellates of the Australian termite Mastotermes darwiniensis: identification of their symbiotic bacteria and cellulases. Symbiosis $\mathbf{4 4}$ 51-66 (2007)

Alliegro, M. C. \& Alliegro, M. A. Analysis of centrosome-associated RNA reveals a unique family of genes. Proc. Natl Acad. Sci. USA (in the press).

2. Alliegro, M. C., Alliegro, M.A. \& Palazzo, R. E. Centrosome-associated RNA in surf clam oocytes. Proc. Natl Acad. Sci. USA 103, 9034-9038 (2006) 3. Chapman, M. J. \& Alliegro, M. A. A symbiotic origin for the centrosome? Symbiosis 44, 23-32 (2007).

Wier, A. M., MacAllister, J. \& Margulis, L. Hibernacular behavior of spirochetes inside membrane-bounded vesicles of the termite protist Staurojoenina assimilis. Symbiosis 44, 75-84 (2007) Oklamoto, N. \& Inouye, I. A secondary symbiosis in progress (Hatena). Science 310, 287 (2005).

16. Margulis, L. Symbiosis in Cell Evolution: Microbia Communities in the Archean and Proterozoic Eons 2nd edn (W. H. Freeman, New York, 1993)

Knoll, A. H. in Life on a Young Planet Chs 6,9 (Princeton Univ. Press, Princeton, 2003).

18. Meyer, J. [FeFe] hydrogenases and their evolution: a genomic perspective. Cell Mol. Life Sci. 64, 10631084 (2007)

19. Hackstein, J. H. P. \& Yarlett, N. in Molecular Basis of Symbiosis (ed. Overmann, J.) 117-142 (Springer Berlin-Heidelberg, 2005). 